\title{
Effects of noise letters upon the identification of a target letter in a nonsearch task*
}

\author{
BARBARA A. ERIKSEN and CHARLES W. ERIKSEN \\ University of Illinois, Champaign/Urbana, Illinois 61820
}

\begin{abstract}
During a 1-sec tachistoscopic exposure, Ss responded with a right or left leverpress to a single target letter from the sets $H$ and $K$ or $S$ and $C$. The target always appeared directly above the fixation cross. Experimentally varied were the ty pes of noise letters (response compatible or incompatible) flanking the target and the spacing between the letters in the display. In all noise conditions, reaction time (RT) decreased as between-letter spacing increased. However, noise letters of the opposite response set were found to impair RT significantly more than same response set noise, while mixed noise letters belonging to neither set but having set-related features produced intermediate impairment. Differences between two target-alone control conditions, one presented intermixed with noise-condition trials and one presented separately in blocks, gave evidence of a preparatory set on the part of Ss to inhibit responses to the noise letters. It was concluded that $\mathrm{S}$ cannot prevent processing of noise letters occurring within about $1 \mathrm{deg}$ of the target due to the nature of processing channel capacity and must inhibit his response until he is able to discriminate exactly which letter is in the target position. This discrimination is more difficult and time consuming at closer spacings, and inhibition is more difficult when noise letters indicate the opposite response from the target.
\end{abstract}

A popular experimental approach to the study of visual information processing has been to employ a display in which a target is embedded among a number of noise elements. Typically, the target has been a letter embedded in a display containing other letters. Initially, a major concern of this research was whether the search process itself was serial or parallel in nature. However, it soon became apparent that many important questions centered around the effects of the noise stimuli.

As would be expected, accuracy or speed in identifying the target was found to be dependent upon the similarity of the target and noise (McIntyre, Fox, \& Neale, 1970; Estes, 1972). Reaction time (RT) to the target increased, and probability of its detection decreased as the number of noise elements increased (Estes, 1972). In attempts to understand how these noise variables entered into the search task, it became necessary to control for such variables as the retinal locus of the noise and the target and the spacing between the target and the closest noise letters. Questions were raised as to the extent to which noise letters were processed, and the possibility of shared feature analyzers or detectors was considered.

In spite of repeated investigations of the above parameters, the nature of the search process and the effects of noise elements is far from resolved (Estes, 1972; Kinchla, 1974; Gardner, 1973; Shiffrin \& Gardner, 1972). At this point in the research effort it would appear fruitful to modify the experimental task. To understand the search task and the effect of noise on

\footnotetext{
*This investigation was supported by U.S. Public Health Service Research Grant MH-1206 and U.S. Public Health Service Research Career Program Award K6-MH-22014.
}

target identification, we need an anchor or baseline condition, i.e.; what is the effect of noise on speed or accuracy in target identification when no visual search is required? Can a letter that always appears in the same known location in the visual field be identified as rapidly when surrounded by noise letters as when it appears alone? The search task by definition requires some processing of noise in order to locate the target. Thus, inferences as to the effects of noise are confounded with assumptions as to how this search of the display is carried out.

There is strong evidence that even when search is eliminated, noise letters or other stimuli impair the processing of the designated target letter. Eriksen and his associates (Eriksen \& Hoffman, 1972, 1973; Colegate, Hoffman, \& Eriksen, 1973) have employed circular displays in which an indicator line is presented to the $S$ at various intervals before the target letter and the remainder of the display appears. The indicator clearly designates to the $\mathrm{S}$ the location of the target letter. While this research has been focused upon the nature of selective attention that is involved, there have been three major findings that are directly relevant to the visual search task and the effects of noise upon target location and identification. First, attentional selectivity is unable to eliminate completely the effects of extraneous stimuli. Even when the $\mathrm{S}$ is provided with an indicator as much as $400-500 \mathrm{msec}$ before the target and the noise elements appear (ample time to process the indicator information), the presence of noise letters in the display delays RT to the target letter. Second, the spatial proximity of noise letters to the target has a nonlinear effect upon target RT. Noise letters within $1 / 2$ deg of 
Table 1

Experimental Conditions and Representative Displays

\begin{tabular}{lllllllll}
\hline \multicolumn{1}{c}{ Condition } & \multicolumn{5}{c}{ Example } \\
\hline 1 & Noise Same as Target & H & H & H & $H$ & H & H & H \\
2 & Noise Response Compatible & K & K & K & $H$ & K & K & K \\
3 & Noise Response Incompatible & S & S & S & $H$ & S & S & S \\
4 & Noise Heterogeneous-Similar & N & W & Z & $H$ & N & W & Z \\
5 & Noise Heterogeneous-Dissimilar & G & J & Q & $H$ & G & J & Q \\
6 & Target Alone & & & & H & & & \\
\hline
\end{tabular}

visual angle of the target produce a much greater impairment in RT than those further removed. In fact, once the noise elements are separated by a degree of visual angle from the target, further separation appears to make little if any difference. Third, the effect of noise letters on target RT is predominantly on the response side as opposed to the processing side. If noise letters have the same prior learned response as the target letter but are physically quite different, little impairment in RT to the target letter is obtained (Eriksen \& Hoffman, 1973). On the other hand, if the noise letters require a response opposite or incompatible to that of the target letter, a large impairment in RT is obtained. This finding would tend to place the locus of the effect in terms of response competition.

The above findings suggest that visual attention is not capable of infinitely fine selectivity. Rather, there is a minimal channel size in terms of capacity for simultaneous processing with a capacity exceeding that required for identifying a single letter. Further, the unutilized capacity cannot be shut off and, if there are other letters or stimuli present, they will be processed simultaneously along with the target. In other words, the channel is filled up to its minimal capacity if material is present to fill it. The result is that the $S$ has available 2 , 3 , or perhaps 4 or more letters that complete processing at essentially the same time and begin the process of evoking responses.

But at this point the information processing system experiences a severe limitation. Only one letter at a time can be vocalized overtly or covertly, and a lever cannot be moved both right and left at the same time. Therefore, a selection process is required to determine which stimulus will evoke a response and which stimuli will be inhibited. This selection and inhibition requires a finite period of time which adds to the RT in target identification or detection.

It is here that the spatial proximity effect can be accounted for. In selecting which of several available letters to emit as a response, the $S$ must perform the additional task of determining which one of these letters came from the indicated position. In essence, a spatial discrimination is required. As is true of discrimination in general, small stimulus differences require longer decision times. But the RT-stimulus difference magnitude function becomes asymptotic rather quickly as the stimulus differences increase. Once stimulus differences achieve some minimal value, further increases are not detectable in terms of further reductions in RT. In terms of the spatial proximity effect found with circular displays, a separation between noise letters and target of greater than approximately $1 / 2 \mathrm{deg}$ produces little or no further decrease in RT.

To recapitulate, the $\mathrm{S}$ in an experiment using circular displays with a leading indicator experiences an indicator which directs attention to a particular position in the display. However, the minimal capacity of his visual attentional channel is such that he will process two or more letters from that sector of the display. He thus has available essentially simultaneousiy from the processing side of the chain several letters that start to evoke or carry through as responses. An inhibitory process is required to prevent the responses from running off willy-nilly. During this inhibitory period, a selection must be made from the various alternative stimuli that have been processed. In the case of the indicator display, this selection is made in terms of determining which of the available letters matches the indicated position on the display. This selection is more rapid if the spatial discrimination is easy.

\section{METHOD}

\section{Design and Rationale}

In the present experiment, visual search was eliminated by having the target letter always appear in the same location, which was $1 / 2$ deg above the fixation point. $S$ was thoroughly instructed to respond only to the letter in this location and to ignore any and all other letters that might appear simultaneously with the target. The $S$ was required to press a lever to the right (or left) if the target letter was an $\mathrm{H}$ or a $\mathrm{K}$ and in the opposite direction for an S or a C. Except for two control conditions in which the target letter appeared alone in the display, the target was always flanked on either side by three noise letters. There were five conditions of noise: (1) noise identical to target-the target letter was flanked on either side by three repetitions of the target letter; (2) noise same response as target-the target letter was flanked on either side by three repetitions of the letter that was the other member of that target set; (3) noise other target set-target flanked by three occurrences of one of the letters of the opposite response set; (4) noise similar to target-target flanked by letters having features similar to the target set as determined by the Gibson system (Gibson, 1969) but excluding the two letters that were members of the target set; (5) noise dissimilar to target-target flanked by letters having features dissimilar to target set as determined by the Gibson system and excluding the target set. In Table 1 , the conditions are defined with the examples of the corresponding displays in which the target letter in all cases is an "H." Table 2 presents the noise letters used for the similar and dissimilar noise conditions and compares their similarity to the letters in the two target sets in terms of the Gibson system.

A second experimental manipulation concerned the spacing between the adjacent letters in the display. All five noise conditions were run under each of three different spacings. In the closest spacing, the nearest contours of adjacent letters were separated by approximately $.06 \mathrm{deg}$ of visual angle, a distance corresponding to the spacing of letters within a printed word. Spacings of $1 / 2 \mathrm{deg}$ and $1 \mathrm{deg}$ of visual angle were also employed.

During the experimental sessions, data were collected for two control measures. Both involved RT to target letters when no noise letters were present in the display. In the target-alone mixed control, the single-letter display was presented during 
blocks of trials in which all five experimental conditions were represented. In the single-letter blocked control, RTs to the target alone were obtained with the single-letter displays presented in blocks of 12 trials, during which the $S$ knew that the displays containing noise elements would not appear.

In terms of our analysis of the processes involved in this experimental task, we anticipated that faster RTs would be obtained using the blocked trials. When single-letter displays are randomly intermixed with noise letter displays, the $S$ will still be prepared to inhibit responses to noise letters that are processed along with the target. This inhibition must continue until $S$ verifies which letter occurred in the target position. In the blocked trials, $S$ can abandon the inhibitory process, since no noise letters are present and no position verification is required. It is also possible that if letter identification occurs by subanalysis or subprocessing of features, then $S$ could reduce his criterion for a response, basing it upon fewer features.

With respect to the experimental conditions, we anticipated that the longest RTs would be obtained for the condition in which noise letters consisted of repetition of a letter from the opposite target set. If one or more of the noise letters were processed simultaneously with the target, then competing responses would tend to be evoked, requiring not only inhibition but maximum care in position verification. This condition should show rather marked improvement in RT as a spacing between the letters becomes greater and therefore the spatial discrimination easier. Similarly, if the effect of noise letters is primarily due to competition for a single response channel, then minimal effects of noise should be obtained for the identical and same response noise conditions. Since in both of these conditions noise letters are eliciting the same response as the target letter, we anticipated little or no difference between them. If a significant portion of the effect of noise on target identification is attributable to the processing of the stimuli, then differences between the identical and same response noise might be anticipated. If we think in terms of feature detectors, the identical noise would be competing for the same feature detectors which might be expected to slow the processing of the target. On the other hand, the same response noise would be using somewhat different feature detectors, although having some in common. Both conditions would require position verification on the part of $S$, since he needs to verify one of the letters he has available as appearing in the target position. Thus, both conditions should show an improvement in RT as the spatial position variable becomes more discriminable.

The noise-similar and noise-dissimilar conditions are of special interest. If letter recognition occurs by a feature-analyzing process which, in turn, corresponds to Gibson's classification system, we might anticipate that the noise-similar condition would produce longer RTs than the dissimilar condition due to competition with the target for feature analyzers. On the other hand, a process of stimulus generalization would seem to predict that similar features would facilitate the response to the target letter, since the features of the noise letters would tend to elicit the same response as the target.

\section{Subjects}

The six Ss (three male) were paid volunteers, all of whom were graduate or undergraduate students at the University of llinois. All had normal or corrected to normal vision and were right-eye dominant and right-handed.

\section{Apparatus and Stimuli}

Stimuli were presented in a Scientific Prototype Model GA three-field tachistoscope in which the original lamps had been replaced with Sylvania F4T5CWX bulbs. Luminance of all fields was maintained at $10 \mathrm{fL}$, as measured by a Spectra brightness spot meter. Onset of the stimulus was initiated by $S$, using a handswitch in his left hand, which also started a Hunter Model 1522 digital Klockounter. A handrest to the right of S contained a small lever switch which $S$ could press either to the
Table 2

Letter Features as Rated by the Gibson System*

\begin{tabular}{|c|c|c|c|c|c|c|c|c|c|c|}
\hline \multirow[b]{2}{*}{ Features } & \multicolumn{5}{|c|}{$\mathrm{H} \& \mathrm{~K}(\mathrm{NWZ})$} & \multicolumn{5}{|c|}{$\mathrm{S} \& \mathrm{C}(\mathrm{GJQ})$} \\
\hline & $\mathbf{H}$ & $\mathbf{K}$ & $\mathrm{N}$ & W & $\mathrm{Z}$ & $S$ & $\mathrm{C}$ & G & $\mathrm{J}$ & Q \\
\hline \multicolumn{11}{|l|}{ Straight } \\
\hline Horizontal & + & & & & + & & & + & & \\
\hline Vertical & + & + & + & & & & n & & & \\
\hline Diagonal / & & + & & + & + & & & & & \\
\hline Diagonal $\backslash$ & & + & + & + & & & & & & + \\
\hline \multicolumn{11}{|l|}{ Curve } \\
\hline Closed & & & & & & & & & & + \\
\hline Open V & & & & & & & & & + & \\
\hline Open $\mathrm{H}$ & & & & & & + & + & + & + & \\
\hline Intersection & + & + & & & & & & & & + \\
\hline \multicolumn{11}{|l|}{ Redundancy } \\
\hline Cyclic Change & & & & + & & + & & & & \\
\hline Symmetry & + & & & + & & & 4 & & & \\
\hline \multicolumn{11}{|l|}{ Discontinuity } \\
\hline Vertical & + & + & + & & & & & & & \\
\hline Horizontal & & & & & + & & & & & \\
\hline \multirow[t]{2}{*}{ Totals } & 5 & 5 & 3 & 4 & 3 & 2 & 2 & 2 & 2 & 3 \\
\hline & & & & 10 & & & & & 7 & \\
\hline
\end{tabular}

${ }^{*}$ Gibson, 1969 , p. 88

right or left upon identification of the target stimulus. Pressing of the lever also stopped the Klockounter. A pair of lights below the Klockounter indicated to $E$ whether $S$ had pressed the switch in the correct direction for the trial. RT was recorded in milliseconds.

Target stimuli were the capital letters H, K, S, and C. For the conditions employing noise letters having angular features, the letters used were $N, W$, and $Z$. The curved-feature letters were $G$, $J$, and $Q$. All letters were capitals from Paratipe 18-point Futura Bold style. Since Fields 2 and 3 of the tachistoscope were used for the target and noise letters, respectively, and these fields reverse the image, the letters were placed on transparent vinyl cards which were inserted backwards into the holders. White vinyl cards installed in the holders provided the necessary contrast for the displays.

The cards containing the target letters each had a single letter which appeared centered $1 / 2$ deg of visual angle above the fixation cross. On the noise-letter cards, all three noise letters appeared on either side of the target. Three different orders were used for each set so that each letter appeared in each possible position in relationship to the target letter. In addition, noise-letter cards were constructed using each target letter repeated six times. Three different spacings of the noise letters in relationship to the target letter and each other were also employed. For the closest spacing, the letters were placed as if printed material were being constructed. The average between letter distance for this spacing was approximately $.0625 \mathrm{deg}$ of visual angle at the viewing distance of $48 \mathrm{in}$. Between letter (edge to edge) spacings of $1 / 2 \mathrm{deg}$ and $1 \mathrm{deg}$ of visual angle were employed in the other two spacing conditions. Fixation was provided by a $1 / 8-\mathrm{deg}$ cross on a white-backed transparent card in Field 1.

\section{Procedure}

Ss were instructed to hold the handswitch for initiating the stimulus presentation in the left hand and to place the right hand on the handrest with thumb and forefinger holding the response lever. They were told to be sure the fixation cross was in good focus before initiating a trial. Three of the Ss were instructed to press the lever to the right if the target letter was an $H$ or a $K$ and left for an $\mathrm{S}$ or a $\mathrm{C}$. The other three Ss received the opposite instructions. The target always appeared directly above the fixation point. Particular care was taken to insure that $S$ understood that the target always appeared in the same location 


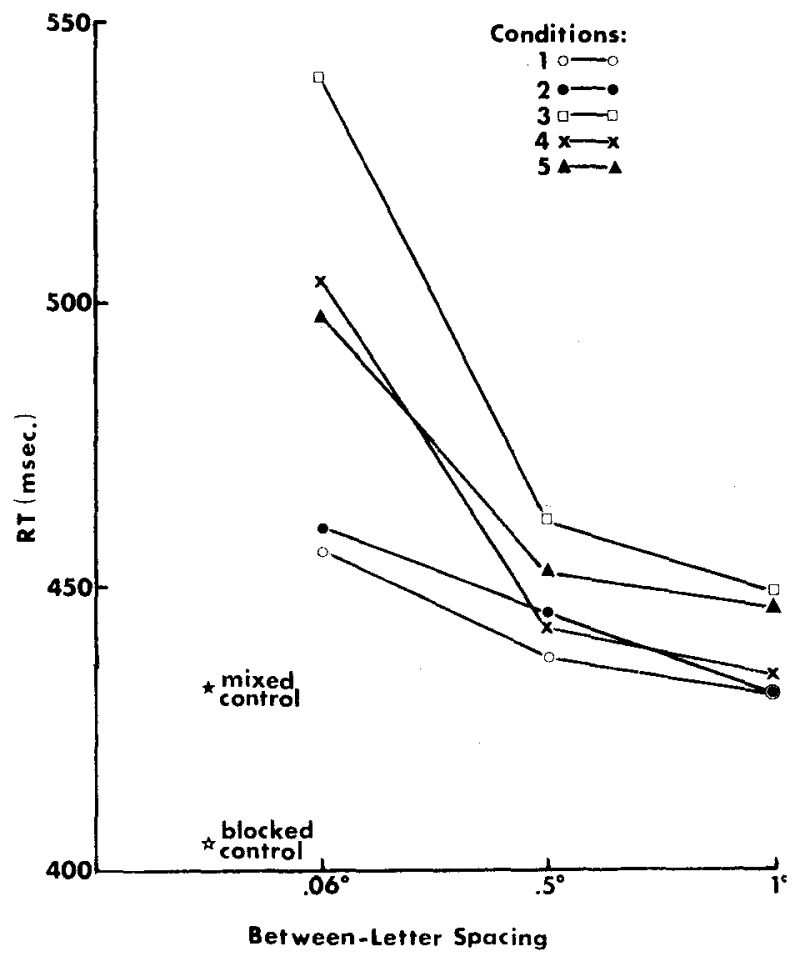

Fig. 1. Mean reaction times (RTs) as a function of spacing (six Ss combined) for the five experimental conditions and two control conditions. Experimental conditions are as follows: (1) noise same as target; (2) noise response compatible; (3) noise response incompatible; (4) noise heterogeneous similar; (5) noise heterogeneous dissimilar.

and that this was the only letter he was to respond to. Viewing was monocular with the right eye. The fixation field went off and the target and noise letters appeared simultaneously as $S$ pressed the handswitch button. They remained on for $1 \mathrm{sec}$ and terminated together, at which time the fixation field came back on. Two small lights, visible only to the $E$, indicated the direction in which the lever had been pressed. Any trials in which $\mathbf{S}$ pressed the lever in the wrong direction or both directions were not recorded and were rerun later in the session. A note was made of the error.

All combinations of conditions by spacing were randomly intermixed within blocks of 32 trials. Four trials in each block contained the target letter alone. An additional block of 12 trials in the single-letter alone condition was run during each session. For experimental presentation, Conditions 4 and 5 (see Table 1) were subdivided as follows: $4 a$-curved target letters ( $S$ and $C$ ) with similar noise (G, J, and $Q) ; 4 b$-angular target letters ( $H$ and $\mathrm{K})$ with similar noise (N, W, and $\mathrm{Z}$ ); 5a-curved target letters (S and $C$ ) with dissimilar noise $(\mathrm{N}, \mathrm{W}$, and $\mathrm{Z}$ ); and $5 \mathrm{~b}$-angular target letters ( $H$ and $K$ ) with dissimilar noise $(G, J$, and $Q$ ).

Each $S$ participated in two practice sessions before beginning the experiment. Six experimental sessions were run, each consisting of three blocks of 32 trials, plus one block of 12 single-letter trials. This yielded a total of 24 trials per $S$ for each condition by spacing combination plus 18 trials per $S$ for each single letter in each of the two control conditions (mixed and blocked).

\section{RESULTS}

The RT data were first analyzed in a three-way analysis of variance (Ss by Spacing by Noise Conditions).
The effects for spacing and noise conditions were both significant beyond the .0001 level with Fs of 58.28 , df $=$ 2,10 , and $\mathrm{F}=28.23$, df $=4,20$, respectively. The $\mathrm{S}$ variable was significant at the .05 level. There was also a significant Noise Condition by Spacing interaction $[\mathrm{F}(8,40)=16.08]$.

The nature of the results can be seen in Fig. 1, where mean RT across Ss is plotted as a function of spacing for each of the five noise conditions. All five noise conditions showed decreasing RT as separation between the display letters increases from .06 to $1 \mathrm{deg}$ of visual angle. The Spacing by Conditions interaction reflects the fact that the difference between conditions is greatest at the closest spacing. When the display letters have been separated by $1 \mathrm{deg}$ of angle, performance for the noise-identical, noise-same-response, and noise-similar conditions is essentially the same. Over all values of the spacing variable, RT to the condition in which the noise was a letter of the opposite response set was the longest, while the fastest RTs were obtained when the noise was identical to the target. There is little difference between this latter condition and the condition in which the noise letter was the other member of the same target set (calling for the same overt response). The lack of a significant difference between these two conditions is attested to by the finding that half of the Ss were faster under the noise-same-response condition than they were when the noise was identical to the target.

With the exception of the closest spacing, the dissimilar-noise condition, in which the noise letters exhibited features similar to letters of the opposite target set, gave longer RTs than when the noise letters contained features similar to the target.

When the letters of the display are separated by $1 \mathrm{deg}$ of visual angle, the noise-similar, noise-identical, and noise-same-response conditions yield RTs that are essentially the same as those obtained for trials in which the target letter alone is presented in blocks of trials containing the other noise conditions. However, this mean RT value is appreciably above that obtained for the target letter alone in blocked trials. The difference is approximately $30 \mathrm{msec}$. The difference between the two target-letter-alone conditions was tested in a three-way analysis of variance (Ss by Target Letter by Mixed vs Blocked Trial Presentation). The effect for mixed vs blocked trials was significant $[F(1,5)=42.38, p<.01]$, as was that for $\mathrm{Ss}[\mathrm{F}(5,3)=20.87, \mathrm{p}<.05]$. There was also a significant $\mathrm{Ss}$ by Letter interaction $[\mathrm{F}(15,15)=$ $7.59, \mathrm{p}<.001]$, reflecting the fact that all Ss tended to make the lever response to the right somewhat faster. There was no significant effect attributable to RTs to the different target letters in the response sets.

The data for the noise-similar and noise-dissimilar conditions were also analyzed separately in a three-way analysis of variance (Ss by Spacing by Conditions). Both the spacing and noise variables were significant at or beyond the .01 level $[F(2,10)=55.32$ and $F(1,5)=$ 15.34, respectively]. The interaction between these two 
Table 3

Errors Per Noise Condition: Six Ss Combined

\begin{tabular}{|c|c|c|c|c|c|c|c|}
\hline \multirow[b]{2}{*}{ Condition } & & \multicolumn{2}{|c|}{$.06 \mathrm{Deg}$} & \multicolumn{2}{|c|}{$1 / 2 \operatorname{Deg}$} & \multicolumn{2}{|c|}{1 Deg } \\
\hline & & $\begin{array}{l}\text { Number } \\
\text { of Errors }\end{array}$ & $\begin{array}{l}\text { Per- } \\
\text { cent }\end{array}$ & $\begin{array}{l}\text { Number } \\
\text { of Errors }\end{array}$ & $\begin{array}{l}\text { Per- } \\
\text { cent }\end{array}$ & $\begin{array}{l}\text { Number } \\
\text { of Errors }\end{array}$ & $\begin{array}{l}\text { Per- } \\
\text { cent }\end{array}$ \\
\hline $\begin{array}{l}\text { Noise Homogeneous } \\
\text { Target Opposite Response Set }\end{array}$ & & 31 & 21.5 & 24 & 16.6 & 6 & 4 \\
\hline $\begin{array}{l}\text { Noise Homogeneous } \\
\text { Target Other Member of Same Response Set }\end{array}$ & $\begin{array}{l}N= \\
144\end{array}$ & 1 & $<1$ & 4 & 2.7 & 6 & 4 \\
\hline Noise $=$ Target & & 6 & 4 & 5 & 3.4 & 4 & 2.7 \\
\hline $\begin{array}{l}\text { Noise Heterogeneous } \\
\text { Target Features Opposite Response Set }\end{array}$ & $N=$ & 34 & 11.8 & 15 & 5.2 & 10 & 3.5 \\
\hline $\begin{array}{l}\text { Noise Heterogeneous } \\
\text { Target Features Same Response Set }\end{array}$ & 288 & 20 & 6.9 & 5 & 1.7 & 18 & 6.3 \\
\hline
\end{tabular}

variables was significant beyond the .05 level $[F(2,10)=$ 4.83].

The response errors were also examined. For the two control conditions, target letter alone-mixed had a 5\% error rate as contrasted with a 7\% error rate for target alone in blocked trials. This does not appear to be a sufficient difference to attribute the superiority of the blocked trials to a speed-accuracy tradeoff. In fact, for one $S$ who made only one error on all target-alone mixed trials and two errors on target-alone blocked trials, there was still a large difference between the two in RT: an average RT of $423 \mathrm{msec}$ on the mixed and $405 \mathrm{msec}$ on the blocked.

A breakdown of the error data for the different noise conditions is presented in Table 3. These error data reflect essentially the same differences between noise conditions and the effect of the spacing variable as was obtained for the RT measures. It is readily apparent that noise of the opposite response set not only slowed Ss' responses but caused them to err much more frequently at the two closest spacings. Similarly, noise consisting of letters having features similar to those of the other target set tended to have much the same effect.

\section{DISCUSSION}

It is quite clear from the above data that even when an $S$ is not required to search for a target letter in a multiletter display, the presence of other letters slows his RT to identification of a target letter. Further, they exert a retarding effect upon RT even when the precise location of the target letter is known to $S$ and the target's position in the visual field is favored over the noise letters in terms of its location on the fovea. Thus, the effect of noise letters upon target identification is not something that is inherent in the search process. However, the question remains as to how this noise introduces its effects upon the idenfication process.

From the present data, it is apparent that the effect of noise is not at some gross level. It is not a sort of "distraction effect," nor a primitive perceptual process that involves the perception of a whole before its parts can be analyzed, nor a rudimentry noting of the presence or absence of items in the visual field. The possibility of rather gross effects such as these upon input processing can be discarded because the delaying effect of noise is markedly determined by its response compatibility with the target. In terms of physical characteristics of the stimulus, flanking the target letter by a different letter that has the same learned response as the target would seem to provide as much physical complexity as flanking the target by a letter requiring an opposite response. Yet in only the latter condition is RT to the target materially increased. Since the effect of noise is strongly determined by its response compatibility with the target letter, support is given not only to the conclusion that the effects of noise are the result of response competition or interference, but further that this results from. at least some of the noise stimuli being processed along with the target to the point where they are identified enough to tend to elicit appropriate responses. This conclusion is commensurate with our suggestion, made in the introduction of this paper, that there is a limit to the degree to which a human $\mathrm{S}$ can restrict his visual attentional capacity and that this minimal level is in excess of the capacity required for processing a single letter.

To the extent that one can make a distinction between the input side of stimulus processing and the output or response side, we feel that the present data favor a theory that puts the locus of the effect of noise at the stage of response selection. One finding in the present data that would tend to implicate an effect at the input processing stage is the finding that even response-compatible noise (noise-identical and noise-same-response conditions) gave longer RTs at the closest stimulus spacings compared with the no-noise mixed condition. If one adopted a limited-energy parallel processing model (Rumelhart, 1970), the effect could be attributed to the distribution of the limited energy over a larger number of stimulus elements with an attendant reduction of the speed with which any single element was processed. In this view, even when the noise letters lead to the same response as the target, their presence in the display would require some of the available processing energy, resulting in the slowing of the processing of the target.

A decrement resulting from energy limitations would 
have to be in addition to the observed response interference effect. Such a model would also have to account for the prominent effect we obtained as a function of the spacing between target and noise letters. It is not clear why processing capacity would have to be shared when the stimuli were $.06 \mathrm{deg}$ of angle apart but not when separated by $1 / 2$ or $1 \mathrm{deg}$ of visual angle.

Our interpretation of the obtained spacing effect is that it represents the ease with which a $S$ can make a spatial or location discrimination. If the $S$ is processing, essentially simultaneously, the target letter and one or more noise letters, some form of an inhibitory process has to be activated in order to prevent responses to the noise letters and to permit selection of the response appropriate to the target location. This response selection must be made in terms of the target letter's location in the display. As with discrimination tasks in general, the more discriminable the differences in location in the display, the faster will be the selection process. This pin-pointing of the target's location would be slowest when the spatial separation was quite small. As the spatial separation increased, the decrease in RT for this discrimination would rapidly become asymptotic. Once a difference is big enough, further increases do not speed discrimination RTs. Even on those trials where all the noise responses led to the same response as the target letter, it is reasonable to expect that the $S$ would still have a check operation to verify that the letter to which he was responding was indeed the one in the target location, since it was necessary for him to be prepared on evety trial for any of the noise conditions.

Other possible sources of the spacing effect seem less likely. At the closest spacing, the possibility of contour interference between adjacent letters exists (Flom, Weymouth, \& Kahneman, 1963). If present, its relative contribution would be slight since the spacing variable interacts markedly with the response compatibility of the noise letters, and there is no obvious reason why contour interference between letters should be greater if their responses are incompatible than if they are compatible.

Since the target letter always appeared $1 / 2$ deg above the fixation point, noise letters fell on progressively less acute areas of the fovea as the spacing between the stimuli increased to $1 \mathrm{deg}$ of angle. It is possible that transmission time for fibers in the retina increases for receptors farther from the center of the fovea. Further, Sternberg (1967) has shown that degrading a stimulus leads to an increase in its processing time. While such effects may possibly be involved in the present spacing effect, their contribution would be minimal in view of the marked interaction between spacing and the response compatibility of the noise letters.

Our view that the spacing effect primarily reflects the ease of response selection is supported by the obtained difference between the two control conditions-target alone mixed vs target alone blocked. Response selection in terms of letter position requires that Ss use some type of inhibitory process in the task to prevent responses from occurring until the selection has been made. The presence of such an inhibitory process would account for the differences between these two control conditions. In the mixed control, the target letter appeared without noise but on unannounced trials that occurred during blocks in which all experimental conditions were represented. In this case, when $S$ initiated a trial it would be to his advantage to have inhibitory processes activated, whereas in the blocked condition $S$ knew that all trials in the 12-trial block would consist only of the target letter being presented. RT in that latter condition averaged approximately $30 \mathrm{msec}$ faster than in the other control. Here the $\mathrm{S}$ could have discarded any inhibitory process in preparation for a trial.

An interpretation of noise effects as resulting from interference at the response selection stage requires a closer examination of the results obtained from the noise-similar and noise-dissimilar conditions. At the closest spacings, these two conditions gave RTs midway in the range between same response noise and opposite response noise. At the two wider spacings, similar noise gave RTs that were not reliably different from those obtained to identical or same response noise, while dissimilar noise approached the RTs obtained under opposite response noise condition. That both similar and dissimilar noise have the same effect on RT at the .06-deg spacing is not readily explainable. However, at the two wider spacings, their performance is consistent with what would be expected in terms of stimulus generalization. Similar noise letters had features in common with the target letter, whereas dissimilar noise letters had features in common with the opposite target set. To attribute the effect to stimulus generalization, however, does little more than relate the finding to an established phenomenon in the experimental literature (Guttman \& Kalish, 1956).

The questions of interest at this point are what mediates stimulus similarity and how is a common response facilitated? Bamber (1969) has proposed an "identity detector" based on the physical identity of two stimuli. However, our data indicate that both a physical and functional identity are detected with equal speed.

Estes (1972) and Bjork and Estes (1973) have attributed the effect of noise letters to possible competition between or an inhibition among feature analyzers. Their interpretation places the effects of noise on the input side of processing. A suggestion by Bjork and Estes (1973) that more complex letters with more features produce more lateral masking and are in turn more resistant to lateral masking was not borne out by the present experiment. It is, of course, possible that the strong effect of response conflict obscured any effects of feature complexity. While our interpretation emphasizes the locus of the effect at a response selection stage, it 
does not preclude the possibility of submechanisms such as feature detectors playing a role on input processing. Indeed, such mechanisms as this may be the basis for the stimulus generalization effects.

\section{REFERENCES}

Bamber, D. Reaction times and error rates for "same"."different" judgments of multidimensional stimuli. Perception \& Psychophysics, 1969, 6, 169-174.

Bjork, E. L., \& Estes, W. K. Letter identification in relation to linguistic context and masking conditions. Memory \& Cognition, 1973, 1, 217-223.

Colegate, R. L., Hoffman, J. E., \& Eriksen, C. W. Selective encoding from multielement visual displays. Perception \& Psychophy sics, 1973, 14, 217-224.

Eriksen, C. W., \& Hoffman, J. E. Temporal and spatial characteristics of selective encoding from visual displays. Perception \& Psychophysics, 1972, 12, 201-204.

Eriksen, C. W.. \& Hof fman, J, E. The extent of processing of noise elem ents during selective encoding from visual displays. Perception \& Psychophysics, 1973, 14, 155-160.

Estes, W. K. Interactions of signal and background variables in visual processing. Perception \& Psychophysics, 1972, 12 , 278-286.

Flom, M. C., Weymouth, F. W., \& Kahneman, D. Visual resolution and contour interaction. Journal of the Optical Society of America, 1963, 53, 1026-1032.

Gardner, G. T. Evidence for independent parallel channels in tachistoscopic perception. Cognitive Psychology, 1973, 4, 130-155.

Gibson, E. J. Principles of perceptual learning and development. New York: Appleton-Century-Crofts, 1969.

Guttman, N., \& Kalish, H. I. Discriminability and stimulus generalization. Journal of Experimental Psychology, 1956, 51 , 79-88.

Kinchla, R. Detecting target elements in multielem ent arrays: $A$ confusability model. Perception \& Psychophysics, 1974, 15, 149-158.

McIntyre, C., Fox, R., \& Neale, J. Effects of noise similarity and redundancy on the information processed from brief visual displays. Perception \& Psychophysics, 1970, 7, 328-332.

Rumelhart, D. E. A multicomponent theory of the perception of briefly exposed visual displays. Journal of Mathematical Psychology, 1970, 7, 191-218.

Shiffrin, $R$.. \& Gardner, G. T. Visual processing capacity and attentional control. Journal of Experimental Psychology. $1972,93,72 \cdot 82$.

Sternberg, $\mathbf{S}$. Two operations in character recognition: Some evidence from reaction time measurements. Perception \& Psychophysics, 1967, 2, 45-53.

(Received for publication February 19, 1974; accepted March 27, 1974.) 